\title{
Antidepressant use in children questioned
}

Recent warnings of suicide risk associated with antidepressant use in children have highlighted the lack of understanding of paediatric depression

\section{Simon Frantz, London}

A US Food and Drug Administration (FDA) public health advisory urging caution in prescribing antidepressants to infants and adolescents with depression has reignited debate on the use of these drugs in younger age groups.

The FDA advisory, issued on 27 October, backed down from a previous advisory issued on 19 June, which recommended that the selective serotonin-reuptake inhibitor (SSRI) paroxetine (Paxil/Seroxat) should not be used in patients with major depression who are under 18 years of age, because of an elevated risk of suicidal thoughts and acts.

Similar recommendations to the original advisory had been made by the UK government's Medicine and Healthcare Products Regulatory Agency (MHRA) on 10 June. On 19 September, an Expert Working Group on SSRIs for the Committee on Safety of Medicines, part of the MHRA, concluded that data from trials on the bicyclic antidepressant venlafaxine (Efexor) showed "an increase in the rate of harmful outcomes including hostility, suicidal ideation and self-harm in the [treated] group compared with the placebo group."

Child psychiatrists say that this is not a reflection of the treatments, but more of the condition that the children have. "Around 20\% of children who are diagnosed with major depression will develop bipolar symptoms, which elevates suicide risk," says Floyd Sallee, Professor of Psychiatry at the University of Cincinnati Medical Center, Ohio, USA. However, these symptoms of bipolar disorder do not manifest themselves until much later after diagnosis. "The problem is that we don't know how to predict when or if bipolarity will develop in children," says Robert Findling, Director of Child and Adolescent Psychiatry at University Hospitals of Cleveland in Ohio. By contrast, in adults, any bipolar symptoms will already be apparent at the time of diagnosis of depression.

However, other reasons for these adverse effects in children cannot be ruled out. Depression in children could be different to that in adults, as the brain is rapidly developing into the late teens. An older drug class used in adults - tricyclic antidepressants has been shown to lack efficacy in children.

All of which points to the fact that more information about antidepressants, and juvenile and adolescent depression, is desperately needed. David Bylund, Professor of Pharmacology at the University of Nebraska Medical Center, is trying

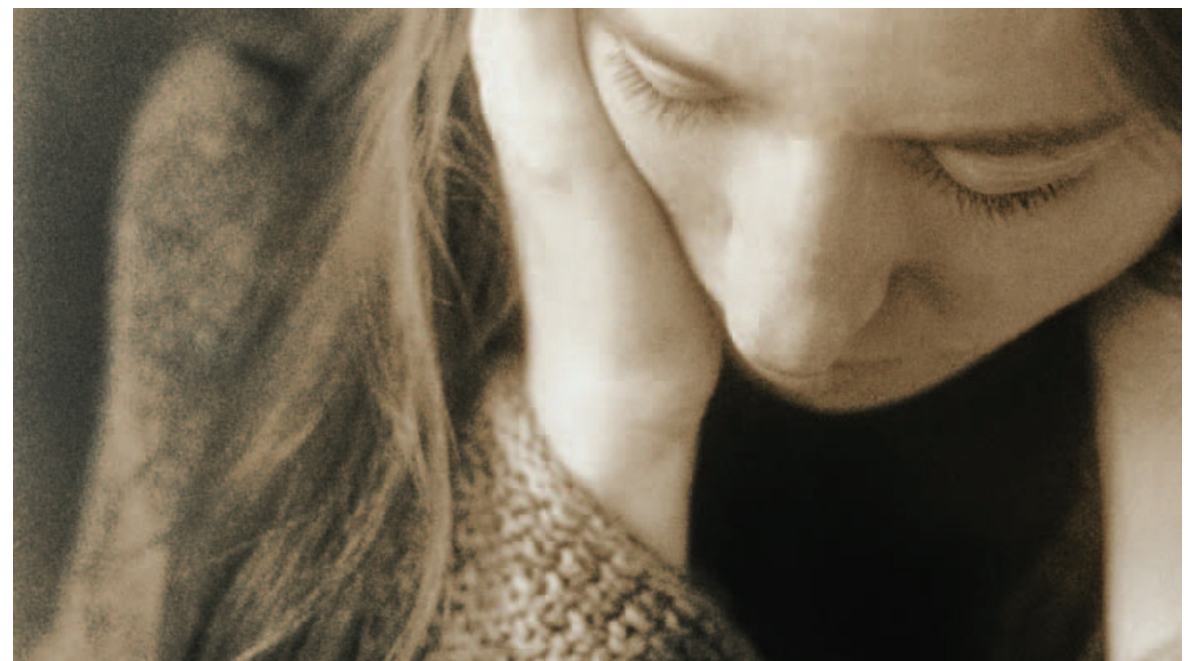

The US FDA is urging caution in prescribing antidepressants to infants and adolescents with depression.

to rectify the lack of preclinical models by developing 'childhood' models from existing adult animal models of depression. "The hope is that we will be able to screen antidepressants like SSRIs and find out more about the differential neurochemistry in these childhood models," he says.

More clinical trials are also needed, but in the case of paediatric trials, this is easier said than done. Around 75\% of approved drugs contain no labelling information for children. Currently, fluoxetine (Prozac) is the only treatment labelled for use in paediatric major depression, with the others being used offlabel. Although the FDA's recent Paediatric Exclusivity provision, which gives companies a six-month extension on their patent if they conduct paediatric trials, has helped, the incentive for companies to test older drugs in new populations is small, with clinicians obtaining dosing regimens from extrapolation of data from adults to children.

\section{"Only when randomized control trials are carried out will we know the true answer."}

What trials have been carried out are difficult to interpret. In its latest advisory, the FDA said it has completed a preliminary review of such reports for the antidepressants citalopram (Celexa/Cipramil), fluoxetine, fluvoxamine (Luvox/Faverin), mirtazapine (Remeron), nefazodone (Serzone), paroxetine, sertraline (Zoloft), and venlafaxine (Efexor), and determined that "the data do not clearly establish an association between the use of these drugs and increased suicidal thoughts or actions by paediatric patients". But it added that "it is not possible at this point to rule out an increased risk of these adverse events for any of these drugs".

One possible reason is that there tends to be an abnormally large placebo-response rate in paediatric trials. This could be because children are more malleable and might respond more favourably to placebo conditions, or because both child and parent discuss the child's symptoms in these trials, which could provide more information than would be collected from an adult trial patient alone.

"Only when randomized control trials are carried out will we know the true answer," says David Healy, Director of the Department of Psychosocial Medicine at the University of Wales, Bangor. Healy and Sallee agree that this discussion is also best served with a more careful scientific re-examination of all data. "In addition, the FDA could request submission of data for group analysis across the SSRI class, so as not to single out one drug, although each study has different design features," says Sallee.

"We're grateful that we have these treatments available, because childhood depression is a major cause of death in teenagers, and the malignancy of the condition is something that is not often mentioned," says Josephine Elia, Associate Medical Director at the Children's Clinical Research Institute, Philadelphia. So, what child psychiatrists urgently need is to understand the biological basis for any potential risks and to conduct more trials, designed more thoroughly, to take these issues into account. 